\title{
Public perception and characteristics related to acceptance of the sugar-sweetened beverage taxation launched in France in 2012
}

\author{
Chantal Julia ${ }^{1,2, *}$, Caroline Méjean ${ }^{1}$, Florence Vicari ${ }^{1}$, Sandrine Péneau ${ }^{1}$ and \\ Serge Hercberg ${ }^{1,2}$ \\ 'Université Paris 13, Sorbonne Paris Cité, Equipe de Recherche en Epidémiologie Nutritionnelle (EREN), Centre \\ d'Epidémiologie et Biostatistiques, Inserm, Inra, Cnam, 74 rue Marcel Cachin, F-93017 Bobigny Cedex, France: \\ ${ }^{2}$ Département de Santé Publique, Hôpital Avicenne (AP-HP), Bobigny, France
}

Submitted 23 April 2014: Final revision received 26 November 2014: Accepted 3 December 2014: First published online 28 January 2015

\begin{abstract}
Objective: In France, an excise tax on sugar-sweetened beverages was introduced on 1 January 2012. Our objective was to assess perception of this tax as well as the sociodemographic characteristics of its supporters and opponents.

Design: Cross-sectional study within the Nutrinet-Santé cohort. A sub-sample of 1996 individuals was selected among participants in the Nutrinet-Santé cohort study. Perceptions of the sugar-sweetened beverage tax were assessed via self-administered questionnaires. The sociodemographic and dietary profiles of supporters and opponents of this tax were explored by multinomial logistic regression.

Setting: Nationally representative French sample, 2012.

Subjects: Adults aged $>18$ years (largest sample $n$ 1996).

Results: Half of the study sample was generally supportive of the tax and $57.7 \%$ perceived it as helpful in improving population health. Participants were more likely to support the tax model if the revenue it generated would be used for health-care system improvement $(72 \cdot 7 \%)$ and if such taxing was associated with a corresponding decrease in the prices of other foodstuffs $(71.5 \%)$. Older participants were more likely to support the tax than were their younger counterparts $(\mathrm{OR}=2 \cdot 37$; 95\% CI 1.60, 3.49 for $>65$ years $v$. 26-45 years; $P<0 \cdot 001)$. Participants with lower educational levels were less likely to support the tax than were those with more formal education (OR $=0.31 ; 95 \%$ CI $0 \cdot 19,0.52$ for low educational level $v$. high education level; $P<0 \cdot 001)$. In our models, sugarsweetened beverage consumption was not associated with tax perception.

Conclusions: The French sugar-sweetened beverage tax appeared to be favourably perceived by the public. Sociodemographic factors modulated such perceptions and should thus be taken into consideration when drafting future public health measures.
\end{abstract}

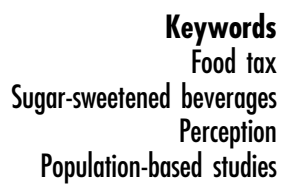

Obesity is rising worldwide and is reaching alarming proportions in developed countries ${ }^{(1)}$. In 2006 in France, $17.9 \%$ of the population was estimated to be obese and an additional $32.4 \%$ to be overweight ${ }^{(2)}$. Poor nutrition and lack of physical activity have been identified as the main predictors of obesity ${ }^{(3,4)}$.

Multiple public health interventions have already tackled this issue, from interventions aiming at improving individual diet via nutritional education to regulation of the food environment ${ }^{(5)}$. Fiscal policies such as taxation of unhealthy foods or beverages have also gained considerable attention the past few years ${ }^{(6-21)}$. Studies have found a small but significant impact of such measures, depending on the type of food and the type (fixed or value-added tax) and level of the taxing scheme $e^{(7,19)}$. Among the various foods or beverages subject to such taxing, sugar-sweetened beverages have been consistently advanced as primary targets ${ }^{(22,23)}$.

The consumption of such products has been recognized as an important risk factor for obesity ${ }^{(24,25)}$. Reducing sweetened beverage consumption among children and adults could therefore contribute to the reduction of the obesity prevalence in industrialized countries ${ }^{(26)}$.

However, the actual effectiveness of fiscal policies depends on their acceptance by the public ${ }^{(16)}$. Regarding 
the taxation of sugar-sweetened beverages, its success also depends on the public's perception of the real purpose and the possible impact in terms of consumption regulation and ultimately health status ${ }^{(16)}$.

Such considerations, however, have not been thoroughly investigated in the literature.

In January 2012, France introduced a new fiscal policy on sweetened beverages (using natural sugars or artificial sweeteners) consisting of an excise tax of $7 \cdot 16 €$ per 100 litres ${ }^{(27)}$.

We investigated the acceptance and perceptions of such a taxation scheme nine months after its launch. We used a representative sample of the French population, enrolled in the Nutrinet-Santé cohort study. We investigated the cross-sectional associations of tax perception with sociodemographic factors and actual sweetened-beverage consumption. We hypothesized that demographic and socio-economic factors as well as sugar-sweetened beverage consumption would be independently associated with acceptance of the tax scheme.

\section{Methods}

\section{Population}

Participants were selected from the Nutrinet-Santé cohort, a prospective observational study in which inclusion and follow-up take place online using a dedicated and secure website (www.etude-nutrinet-sante.fr). The main objectives of the Nutrinet-Santé study are to: (i) investigate the relationships between nutrition and health; and (ii) elucidate the role of various determinants of individual dietary patterns and nutritional status, and their interactions. Inclusion in the study began in May 2009 and is still ongoing. By March 2012, more than 120000 individuals were included in the cohort. Detailed information about the design/methods and rationale of the Nutrinet-Santé study can be found elsewhere ${ }^{(28)}$.

Briefly, volunteers from the general population aged $>18$ years are considered actively enrolled in the cohort upon completion of a set of questionnaires assessing diet (via three dietary records randomly distributed over a 2-week period), physical activity, anthropometrics, lifestyle, socio-economic conditions and health status. Once the individuals are included in the study, they receive additional questionnaires on a monthly basis on specific topics pertaining to dietary behaviours, determinants of eating habits and health status. Six months after enrolment, they receive a validated semi-quantitative $\mathrm{FFQ}^{(29)}$.

A sub-sample of 5000 individuals representative of the French population with respect to sex, age and occupational category ${ }^{(30)}$ was selected among participants in the NutrinetSanté cohort by a stratified random sampling procedure.

\section{Etbics statement}

The Nutrinet-Santé study is conducted in accordance with the Declaration of Helsinki and all procedures have been approved by the Institutional Review Board of the French Institute for Health and Medical Research (0000388FWA 00005831) and the Commission Nationale Informatique et Libertés (908450 and 909216). Electronic informed consent was obtained from all participants.

\section{Data collection}

\section{Tax perception}

A questionnaire pertaining to various aspects of the attitudes and perception of the tax on sweetened beverages was developed by a multidisciplinary research team, including experts from the fields of economics, nutrition and social sciences. The questionnaire was then administered to the selected sub-sample and was available for a 6-month period, 9 to 15 months after the launch of the sugar-sweetened beverage tax (i.e. from 7 September 2012 to 7 March 2013). Median response time was $5 \mathrm{~d}$.

Questionnaire items pertained to issues regarding acceptance of the tax ('I support imposing a tax on sweetened beverages', 'I support imposing taxes on any and all foods and beverages which are bad for health', 'I support imposing a tax on sweetened beverages only if the money is then used to improve the health-care system'), perceptions of its economic impact ('Imposing a tax on sweetened beverages would increase prices and reduce purchasing power', 'A tax on sweetened beverages would be unfair because poor people would still need to pay the same amount as the rich', 'I support imposing a tax on sweetened beverages only if the prices of other foods and beverages (which are good for health) go down') and its potential impact on health ('Having a tax on sweetened beverages would help improve population health'). Perception-related items were all positively phrased in the active voice, with 5-point Likert scales as responses from 'I strongly agree' to 'I strongly disagree', plus 'I don't know'.

Attitudes about price modification and knowledge of the taxation and its scope were also investigated.

\section{Demographic and socio-economic data}

Such data were collected at inclusion, through selfadministered questionnaires, and included age, gender, education (no diploma and primary, secondary, postsecondary graduate), number and age of children living in the household, and income. Income per household unit was calculated using information about household income and composition $^{(30)}$. The following categories of monthly income were used: $<900 €, 900-2700 €$ and $>2700 €$ per household unit. A supplemental category was used for participants refusing to disclose information concerning their income. Household composition was divided as follows: no children, one or more children (aged 0-13 years), one or more adolescents (aged 14-18 years), and children in both age categories. Each variable was selected after a careful review of the literature and reflected a different aspect of the demographic/socio-economic profile of the respondent ${ }^{(31)}$. 


\section{Sweetened beverage consumption}

Data on sweetened beverage consumption were derived from a validated semi-quantitative $\mathrm{FFQ}^{(29)}$, which was administered to all participants in the Nutrinet-Santé study six months after inclusion. The food list contained 240 food and beverage items categorized into twenty-two different categories. Sugar-sweetened and artificially sweetened beverage consumption was assessed via specific items in the questionnaire. Additional questions inquired about type of fat used for cooking and dressing, and about consumed items not appearing on the list. For most food items, participants were asked to report their consumption frequency on the basis of the number of portions consumed. Standard portion sizes were clearly noted using common household measurements (such as a spoon) or familiar unit sizes (such as a cup of yoghurt). The frequency of consumption referred to usual consumption over the past year, assessed in a descending order: yearly, monthly, weekly or daily. Participants were asked to provide only one answer.

\section{Statistical analyses}

Only individuals who had completed the sweetened beverage tax perception questionnaire were eligible for the analyses. We excluded individuals who had systematically answered 'I don't know' to all perception-related questions, those with missing data on sweetened beverage consumption, and those with 'I don't know' responses to individual questions.

Given the response distribution observed in the sample, perception-related variables were grouped into three categories: 'I agree', 'I neither agree nor disagree' (hereafter termed 'neither') and 'I disagree'.

As no breakpoint was observed and given the response distribution observed in the sample, consumption of sugar-sweetened beverages was divided in tertiles. Consumption of artificially sweetened beverages was divided in none $v$. some (i.e. consumers).

Comparisons between included and excluded individuals were undertaken with $\chi^{2}$ tests. Associations between the tax perception variables and the sociodemographic factors were assessed using multinomial regression. Tax perception variables were modelled as dependent variables, with the category 'I disagree' used as the reference category. Sociodemographic data and sweetened beverage consumption were modelled as independent variables. For each outcome, variables were first explored in univariate models (see online supplementary material, Supplemental Table 1, for univariate results) and those with $P<0.2$ were selected for multivariate analyses. Moreover, some variables were forced in the models because they appeared to be important confounding factors, such as sweetened beverage consumption or household composition. Analyses were performed using the SAS statistical software package version 9.3. All tests were two-sided and $P<0.05$ was considered as significant.

\section{Results}

Among the 5000 individuals who received the tax perception questionnaire, 2509 returned completed questionnaires. Among them, eighty-seven had systematically answered 'I don't know' to all perception-related questions and were thus excluded from the analyses. In the remaining sample, 2044 individuals had available data on beverage consumption from the FFQ. Further exclusion of individuals having answered 'I don't know' to any individual questions led to a maximal sample of 1996 participants.

Compared with individuals who had received the sweetened beverage tax perception questionnaire but were not included in the final sample, included individuals were more likely to be older $(12 \cdot 8 \% v .21 \cdot 6 \%$, respectively, were aged $>65$ years; $P<0.001)$, to have a higher educational level $(52.0 \%$ v. $55.0 \%$, respectively, had post-secondary education, $P<0.001)$ and higher income (17.9\% v. $22.5 \%$, respectively, had income $>2700 €$ /month, $P<0.001)$. Sociodemographic characteristics of the included individuals are shown in Table 1.

Overall, the sweetened beverage tax was supported by almost half of the participants (48.5\% were supportive of the tax whereas $31.2 \%$ were not supportive, and $20.3 \%$ did not have an opinion; Table 2 ). This tax was more likely

Table 1 Sociodemographic characteristics of participants included in the present study (largest sample $n$ 1996); a nationally representative sub-sample from the Nutrinet-Santé cohort study, France, 2012

\begin{tabular}{|c|c|c|}
\hline & \multicolumn{2}{|c|}{$\begin{array}{l}\text { Included } \\
\text { participants }\end{array}$} \\
\hline & $n$ & $\%$ \\
\hline \multicolumn{3}{|l|}{ Sex } \\
\hline Women & 1044 & $52 \cdot 3$ \\
\hline Men & 952 & $47 \cdot 7$ \\
\hline \multicolumn{3}{|l|}{ Age } \\
\hline $18-25$ years & 50 & 2.5 \\
\hline $26-45$ years & 633 & $31 \cdot 7$ \\
\hline $46-65$ years & 975 & $48 \cdot 8$ \\
\hline$>65$ years & 338 & $16 \cdot 9$ \\
\hline \multicolumn{3}{|l|}{ Level of education } \\
\hline No diploma and primary & 100 & $5 \cdot 0$ \\
\hline Secondary & 798 & $40 \cdot 0$ \\
\hline Post-secondary graduate & 1098 & $55 \cdot 0$ \\
\hline \multicolumn{3}{|l|}{ Income per household unit } \\
\hline$<900 € /$ month & 173 & $8 \cdot 7$ \\
\hline $900-2700 € /$ month & 1194 & $59 \cdot 8$ \\
\hline$>2700 € /$ month & 450 & 22.5 \\
\hline Refuses to answer & 179 & $9 \cdot 0$ \\
\hline \multicolumn{3}{|l|}{ Household composition } \\
\hline No children & 1535 & $76 \cdot 9$ \\
\hline One or more children (aged $0-13$ years) & 315 & $15 \cdot 8$ \\
\hline One or more adolescents (aged $14-18$ years) & 87 & 4.4 \\
\hline Children of both age categories & 59 & 3.0 \\
\hline \multicolumn{3}{|l|}{ Sweetened beverage consumption } \\
\hline Tertile 1 & 668 & 33.5 \\
\hline Tertile 2 & 662 & $33 \cdot 2$ \\
\hline Tertile 3 & 666 & $33 \cdot 4$ \\
\hline \multicolumn{3}{|l|}{ Artificially sweetened beverages } \\
\hline Non-consumer & 1395 & $69 \cdot 9$ \\
\hline Consumer & 601 & $30 \cdot 1$ \\
\hline
\end{tabular}


Table 2 Global perception of the sugar-sweetened beverage tax scheme among a nationally representative sub-sample (largest sample $n$ 1996) from the Nutrinet-Santé cohort study, France, 2012

\begin{tabular}{|c|c|c|c|c|c|c|c|}
\hline & \multicolumn{2}{|c|}{ Agree } & \multicolumn{2}{|c|}{ Neither } & \multicolumn{2}{|c|}{ Disagree } & \multirow{2}{*}{$\frac{\text { Total }}{n}$} \\
\hline & $n$ & $\%$ & Neither & $\%$ & $n$ & $\%$ & \\
\hline I support imposing a tax on sweetened beverages & 955 & 48.5 & 400 & $20 \cdot 3$ & 615 & $31 \cdot 2$ & 1970 \\
\hline $\begin{array}{l}\text { I support imposing taxes on any and all foods and beverages which are bad } \\
\text { for health }\end{array}$ & 1118 & $56 \cdot 7$ & 328 & $16 \cdot 6$ & 527 & $26 \cdot \overline{7}$ & 1973 \\
\hline $\begin{array}{l}\text { I support imposing a tax on sweetened beverages only if the money is then } \\
\text { used to improve the health-care system }\end{array}$ & 1436 & $72 \cdot 7$ & 275 & 13.9 & 263 & $13 \cdot 3$ & 1974 \\
\hline $\begin{array}{l}\text { I support imposing a tax on sweetened beverages only if the prices of other } \\
\text { foods and beverages (which are good for health) go down }\end{array}$ & 1396 & 71.5 & 257 & $13 \cdot 2$ & 298 & $15 \cdot 3$ & 1951 \\
\hline $\begin{array}{l}\text { Imposing a tax on sweetened beverages would increase prices and reduce } \\
\text { purchasing power }\end{array}$ & 1273 & $63 \cdot 8$ & 320 & $16 \cdot 0$ & 403 & $20 \cdot 2$ & 1996 \\
\hline $\begin{array}{l}\text { A tax on sweetened beverages would be unfair because poor people would still } \\
\text { need to pay the same amount as the rich }\end{array}$ & 967 & $49 \cdot 3$ & 421 & 21.5 & 572 & $29 \cdot 2$ & 1960 \\
\hline Having a tax on sweetened beverages would help improve population health & 1151 & $57 \cdot 7$ & 329 & $16 \cdot 5$ & 515 & $25 \cdot 8$ & 1995 \\
\hline
\end{tabular}

to be favourably perceived if the revenue it generated could be used to improve the health-care system $(72.7 \%$ of support) or if it could be accompanied by a simultaneous decrease in the prices of other foodstuffs $(71.5 \%$ of support). Moreover, the tax on sweetened beverages was perceived as being helpful in terms of the potential to improve population health $(57 \cdot 7 \%$ of support; Table 2$)$.

In multivariable analyses, perceptions of the sweetened beverage tax varied across sociodemographic and dietary characteristics (Tables 3-5). Older participants were more likely to express favourable attitudes towards the tax and to its application to any foods with potential adverse health effects than were younger ones ( $\mathrm{OR}=2 \cdot 37 ; 95 \% \mathrm{CI}$ $1 \cdot 60,3.49$ and $\mathrm{OR}=3 \cdot 15 ; 95 \%$ CI $2 \cdot 10,4 \cdot 72$, respectively, for participants aged $>65$ years $v .26-45$ years, $P<0 \cdot 001$; Table 3). Participants with lower educational levels were less likely to hold favourable attitudes regarding the tax than were those with higher educational levels $(\mathrm{OR}=0.31$; $95 \%$ CI $0.19,0.52$ for participants with up to elementary education $v$. university-level education, $P<0 \cdot 001$; Table 3 ). Consumption of artificial sweeteners was associated with a lower level of support for the tax scheme (OR =0.69; $95 \%$ CI $0.55,0.87$ for consumers $v$. non-consumers, $P=0.006$ ). No significant difference with any sociodemographic or dietary factors was observed regarding support for the tax scheme on the condition that the generated revenue could be used to improve the health-care system (all $P>0.05$; Table 3).

Participants with lower education tended to agree more with the fact that the sweetened beverage tax would deepen social disparities (corresponding to the statement 'A tax on sweetened beverages would be unfair because poor people would still need to pay the same amount as the rich'; $\mathrm{OR}=1.94 ; 95 \% \mathrm{CI} 1.11,3.39$ and $\mathrm{OR}=1.32$; $95 \%$ CI 1.04, 1.66 for participants with up to elementary education and participants with secondary education, respectively, $v$. those with university-level education, $P<0 \cdot 01$; Table 4). Men, those with incomes $<900 € /$ month and those with incomes $>2700 € /$ month were less likely to support the sweetened beverage tax scheme on the condition of a concomitant decrease in the prices of other foodstuffs ( $\mathrm{OR}=0.63 ; 95 \%$ CI 0.48, 0.81 for men $v$. women, $P<0.001 ; \mathrm{OR}=0.62 ; 95 \% \mathrm{CI} 0.40,0.95$ for participants with incomes $<900 € /$ month and $\mathrm{OR}=0.72 ; 95 \%$ CI 0.52, 1.00 for participants with incomes $>2700 € /$ month $v$. those with incomes 900-2700 €/month, $P=0.0011$; Table 4).

Participants older than 65 years were more likely to support the contention that the tax would be helpful in improving population health, whereas participants reporting moderate and high consumption of sweetened beverages were less likely to perceive it that way $(\mathrm{OR}=3.01 ; 95 \% \mathrm{CI}$ $2 \cdot 00,4.52$ for participants aged $>65$ years $v$. those aged 26-45 years, $P<0.001 ; \quad O R=0.75 ; 95 \%$ CI $0.58,0.99$ and $\mathrm{OR}=0.67 ; 95 \% \mathrm{CI} 0.51,0.88$ for those with moderate and high consumption, respectively, $v$. those with low consumption, $P=0 \cdot 055$; Table 5).

\section{Discussion}

Our results show that public perception of food taxes in general and the sugar-sweetened beverage tax in particular is favourable in France, particularly as regards its potential to improve the health of the population. Support and perceptions of the potential impact of the tax in terms of economic burden to consumers or in terms of health varied according to sociodemographic factors. Soft drink consumption (whether sugar-sweetened or artificially sweetened), however, did not appear associated with tax perception.

To our knowledge, the present study is the first one to thoroughly investigate public perceptions of a food or beverage tax, going beyond single measures of public acceptance $^{(32,33)}$. In France, prior to the implementation of the tax, an industry-sponsored poll had indicated somewhat lower acceptance of the tax scheme (i.e. $41 \%)^{(33)}$. Surveyed individuals were also very sceptical as to the potential impact of the measure in terms of reducing 
Table 3 Multivariable associations between sociodemographic and dietary variables and sugar-sweetened beverage tax perception among a nationally representative sub-sample (largest sample $n$ 1996) from the Nutrinet-Santé cohort study, France, 2012: adherence variables

\begin{tabular}{|c|c|c|c|c|c|c|c|c|c|c|c|c|c|c|c|}
\hline & \multicolumn{5}{|c|}{$\begin{array}{l}\text { I support imposing a tax on sweetened } \\
\text { beverages }(n 1970)\end{array}$} & \multicolumn{5}{|c|}{$\begin{array}{l}\text { I support imposing taxes on any and all foods } \\
\text { and beverages which are bad for health } \\
\qquad(n \text { 1973) }\end{array}$} & \multicolumn{5}{|c|}{$\begin{array}{l}\text { I support imposing a tax on sweetened } \\
\text { beverages only if the money is then used to } \\
\text { improve the health-care system ( } n \text { 1974) }\end{array}$} \\
\hline & \multicolumn{2}{|r|}{ Agree } & \multicolumn{2}{|c|}{ Neither } & \multirow[b]{2}{*}{$P$} & \multicolumn{2}{|r|}{ Agree } & \multicolumn{2}{|c|}{ Neither } & \multirow[b]{2}{*}{$P$} & \multicolumn{2}{|c|}{ Agree } & \multicolumn{2}{|c|}{ Neither } & \multirow[b]{2}{*}{$P$} \\
\hline & OR & $95 \% \mathrm{Cl}$ & OR & $95 \% \mathrm{Cl}$ & & OR & $95 \% \mathrm{Cl}$ & OR & $95 \% \mathrm{Cl}$ & & OR & $95 \% \mathrm{Cl}$ & OR & $95 \% \mathrm{Cl}$ & \\
\hline \multicolumn{16}{|l|}{ Sex } \\
\hline Women & 1.00 & Ref. & 1.00 & Ref. & & 1.00 & Ref. & 1.00 & Ref. & & 1.00 & Ref. & 1.00 & Ref. & \\
\hline Men & 1.24 & $1.00,1.54$ & 1.03 & $0.79,1.33$ & 0.09 & 1.33 & $1.07,1.65$ & 1.02 & $0.77,1.35$ & 0.015 & 0.90 & $0 \cdot 69,1 \cdot 18$ & 0.87 & $0 \cdot 62,1 \cdot 23$ & 0.69 \\
\hline \multicolumn{16}{|c|}{ - } \\
\hline $18-25$ years & 0.83 & $0.43,1.62$ & 0.55 & $0.23,1.31$ & & 0.54 & $0.27,1.08$ & 0.59 & $0.26,1.35$ & & 1.04 & $0.43,2.51$ & 0.82 & $0.25,2.63$ & \\
\hline $26-45$ years & 1.00 & Ref. & 1.00 & Ref. & & 1.00 & Ref. & 1.00 & Ref. & & 1.00 & Ref. & 1.00 & Ref. & \\
\hline $46-65$ years & 1.11 & $0.84,1.47$ & 0.91 & $0.65,1.28$ & & 1.37 & $1.03,1.81$ & 0.92 & $0.64,1.32$ & & 1.01 & $0.71,1.43$ & 0.67 & $0.43,1.05$ & \\
\hline$>65$ years & 2.37 & $1.60,3.49$ & 1.44 & $0.90,2.31$ & 0.0002 & 3.15 & $2 \cdot 10,4.72$ & 1.27 & $0 \cdot 75,2 \cdot 17$ & $<0.001$ & 1.63 & $0.99,2.67$ & 1.24 & $0.67,2.29$ & 0.094 \\
\hline \multicolumn{16}{|l|}{ Level of education } \\
\hline No diploma and primary & 0.31 & $0.19,0.52$ & 0.50 & $0.27,0.93$ & & 0.42 & $0.26,0.69$ & 0.54 & $0.28,1.06$ & & 0.70 & $0.39,1.26$ & 1.03 & $0.49,2 \cdot 19$ & \\
\hline Secondary & 0.52 & $0.41,0.66$ & 0.80 & $0.61,1.07$ & & 0.65 & $0.51,0.83$ & 0.87 & $0.64,1.19$ & & 0.97 & $0.72,1.31$ & 0.98 & $0.67,1.44$ & \\
\hline Post-secondary graduate & 1.00 & Ref. & 1.00 & Ref. & $<0.001$ & 1.00 & Ref. & 1.00 & Ref. & 0.0004 & 1.00 & Ref. & 1.00 & Ref. & 0.61 \\
\hline \multicolumn{16}{|l|}{ Income per household unit } \\
\hline$<900 € /$ month & 0.85 & $0.58,1.24$ & 0.93 & $0.59,1.46$ & & 0.87 & $0.59,1.27$ & $1 \cdot 11$ & $0.69,1.79$ & & 0.81 & $0.51,1.30$ & 0.81 & $0.44,1.48$ & \\
\hline $900-2700 € /$ month & 1.00 & Ref. & 1.00 & Ref. & & 1.00 & Ref. & 1.00 & Ref. & & 1.00 & Ref. & 1.00 & Ref. & \\
\hline$>2700 € /$ month & 1.32 & $1.00,1.74$ & 1.03 & $0.73,1.46$ & & 1.18 & $0.89,1.57$ & 0.96 & $0.65,1.41$ & & $1 \cdot 11$ & $0.78,1.59$ & $1 \cdot 12$ & $0.71,1.76$ & \\
\hline Refuses to answer & 0.94 & $0.65,1.36$ & 1.01 & $0.65,1.58$ & 0.37 & 1.13 & $0.76,1.67$ & 1.54 & $0.96,2.45$ & 0.26 & 0.75 & $0.48,1.17$ & 0.78 & $0.43,1.42$ & 0.82 \\
\hline \multicolumn{16}{|l|}{ Household composition } \\
\hline No children & 1.00 & Ref. & 1.00 & Ref. & & 1.00 & Ref. & 1.00 & Ref. & & 1.00 & Ref. & 1.00 & Ref. & \\
\hline One or more children (aged 0-13 years) & 0.71 & $0.52,0.97$ & 0.73 & $0.50,1.07$ & & 0.81 & $0.60,1.11$ & 0.73 & $0.49,1.10$ & & $1 \cdot 18$ & $0.77,1.79$ & 1.44 & $0.86,2.42$ & \\
\hline One or more adoles & 0.86 & $0.53,1.40$ & 0.45 & $0.22,0.94$ & & 0.88 & $0.53,1.48$ & 0.76 & $0.37,1.55$ & & 0.51 & $0.29,0.90$ & 0.99 & $0.48,2.04$ & \\
\hline \multirow{2}{*}{\multicolumn{16}{|c|}{ Sweetened beverage consumption }} \\
\hline & & & & & & & & & & & & & & & \\
\hline Tertile 1 & 1.00 & Ref. & 1.00 & Ref. & & 1.00 & Ref. & 1.00 & Ref. & & 1.00 & Ref. & 1.00 & Ref. & \\
\hline Tertile 2 & 0.91 & $0 \cdot 70,1 \cdot 18$ & 1.01 & $0.73,1.38$ & & 1.08 & $0.83,1.41$ & 1.22 & $0.86,1.74$ & & 1.21 & $0.88,1.68$ & $1 \cdot 12$ & $0.73,1.71$ & \\
\hline Tertile 3 & 0.92 & $0.70,1.19$ & 0.97 & $0.70,1.34$ & 0.94 & 0.98 & $0.75,1.28$ & 1.05 & $0.74,1.49$ & 0.81 & 1.25 & $0.89,1.73$ & 1.22 & $0.80,1.87$ & 0.69 \\
\hline \multicolumn{16}{|l|}{ Artificially sweetened beverages } \\
\hline Non-consumer & 1.00 & Ref. & 1.00 & Ref. & & 1.00 & Ref. & 1.00 & Ref. & & 1.00 & Ref. & 1.00 & Ref. & \\
\hline Consumer & 0.69 & $0.55,0.87$ & 0.77 & $0.58,1.02$ & 0.0064 & 0.96 & $0.76,1.21$ & 0.96 & $0.71,1.31$ & 0.93 & 0.99 & $0.74,1.33$ & 0.95 & $0.65,1.38$ & 0.95 \\
\hline
\end{tabular}

Ref., referent category.

Results from multivariable multinomial regression with all variables included in the model. 
Table 4 Multivariable associations between sociodemographic and dietary variables and sugar-sweetened beverage tax perception among a nationally representative sub-sample (largest sample $n$ 1996) from the Nutrinet-Santé cohort study, France, 2012: perception of the economic impact variables

\begin{tabular}{|c|c|c|c|c|c|c|c|c|c|c|c|c|c|c|c|}
\hline & \multicolumn{5}{|c|}{$\begin{array}{l}\text { Imposing a tax on sweetened beverages } \\
\text { would increase prices and reduce } \\
\text { purchasing power }(n \text { 1996) }\end{array}$} & \multicolumn{5}{|c|}{$\begin{array}{l}\text { A tax on sweetened beverages would be } \\
\text { unfair because poor people would still need } \\
\text { to pay the same amount as the rich ( } n \text { 1960) }\end{array}$} & \multicolumn{5}{|c|}{$\begin{array}{l}\text { I support imposing a tax on sweetened } \\
\text { beverages only if the prices of other foods and } \\
\text { beverages (which are good for health) go down } \\
\text { ( } n \text { 1951) }\end{array}$} \\
\hline & \multicolumn{2}{|r|}{ Agree } & \multicolumn{2}{|c|}{ Neither } & \multirow[b]{2}{*}{$P$} & \multicolumn{2}{|c|}{ Agree } & \multicolumn{2}{|c|}{ Neither } & \multirow[b]{2}{*}{$P$} & \multicolumn{2}{|r|}{ Agree } & \multicolumn{2}{|c|}{ Neither } & \multirow[b]{2}{*}{$P$} \\
\hline & OR & $95 \% \mathrm{Cl}$ & OR & $95 \% \mathrm{Cl}$ & & OR & $95 \% \mathrm{Cl}$ & OR & $95 \% \mathrm{Cl}$ & & OR & $95 \% \mathrm{Cl}$ & OR & $95 \% \mathrm{Cl}$ & \\
\hline \multicolumn{16}{|l|}{ Sex } \\
\hline Women & 1.00 & Ref. & 1.00 & Ref. & & 1.00 & Ref. & 1.00 & Ref. & & 1.00 & Ref. & 1.00 & Ref. & \\
\hline Men & 1.13 & $0.89,1.42$ & 0.73 & $0.54,0.98$ & 0.0032 & 0.84 & $0.68,1.04$ & 0.91 & $0.70,1.18$ & 0.28 & 0.63 & $0.48,0.81$ & $1 \cdot 13$ & $0.80,1.60$ & $<0.001$ \\
\hline \multicolumn{16}{|c|}{ (1) } \\
\hline $18-25$ years & 1.31 & $0.58,2.98$ & 0.91 & $0.31,2.69$ & & 0.88 & $0.45,1.73$ & 0.53 & $0.21,1.35$ & & 1.46 & $0.54,3.95$ & 0.95 & $0.25,3.55$ & \\
\hline $26-65$ years & 1.00 & Ref. & 1.00 & Ref. & & 1.00 & Ref. & 1.00 & Ref. & & 1.00 & Ref. & 1.00 & Ref. & \\
\hline $46-65$ years & 1.27 & $0.94,1.72$ & 0.94 & $0.63,1.41$ & & 1.32 & $1.00,1.75$ & 1.02 & $0.72,1.43$ & & 0.81 & $0.57,1.13$ & 0.80 & $0.51,1.25$ & \\
\hline$>65$ years & 1.28 & $0.86,1.90$ & 1.52 & $0.93,2.50$ & 0.078 & 1.05 & $0.73,1.50$ & 1.14 & $0.75,1.74$ & 0.12 & 1.12 & $0.71,1.79$ & 1.21 & $0.67,2.19$ & 0.45 \\
\hline \multicolumn{16}{|l|}{ Level of education } \\
\hline No diploma and primary & 0.69 & $0.41,1.15$ & 0.51 & $0.24,1.09$ & & 1.94 & $1 \cdot 11,3.39$ & 1.58 & $0.81,3.09$ & & 0.65 & $0.38,1 \cdot 14$ & 0.58 & $0.27,1 \cdot 28$ & \\
\hline Secondary & 1.13 & $0.87,1.46$ & 1.22 & $0.88,1.70$ & & 1.32 & $1.04,1.66$ & 1.27 & $0.96,1.68$ & & 0.87 & $0.66,1.16$ & 0.81 & $0.56,1.18$ & \\
\hline Post-secondary graduate & 1.00 & Ref. & 1.00 & Ref. & $0 \cdot 16$ & 1.00 & Ref. & 1.00 & Ref. & 0.059 & 1.00 & Ref. & 1.00 & Ref. & 0.53 \\
\hline \multicolumn{16}{|l|}{ Income per household unit } \\
\hline$<900 € /$ month & $1 \cdot 15$ & $0.74,1.78$ & $1 \cdot 19$ & $0.67,2.11$ & & 1.08 & $0.72,1.62$ & 0.91 & $0.55,1.51$ & & 0.62 & $0.40,0.95$ & 0.80 & $0.43,1.48$ & \\
\hline $900-2700 € /$ month & 1.00 & Ref. & 1.00 & Ref. & & 1.00 & Ref. & 1.00 & Ref. & & 1.00 & Ref. & 1.00 & Ref. & \\
\hline$>2700 € /$ month & 0.71 & $0.53,0.94$ & 0.96 & $0.66,1.39$ & & 0.68 & $0.52,0.89$ & 0.77 & $0.56,1.06$ & & 0.72 & $0.52,1.00$ & 1.15 & $0.75,1.75$ & \\
\hline Refuses to answer & 1.12 & $0.73,1.72$ & 1.44 & $0.85,2.45$ & 0.12 & 0.64 & $0.44,0.93$ & 0.86 & $0.55,1.33$ & 0.034 & 0.77 & $0.48,1.24$ & $1 \cdot 71$ & $0.96,3.05$ & 0.0011 \\
\hline \multicolumn{16}{|l|}{ Household composition } \\
\hline No children & 1.00 & Ref. & 1.00 & Ref. & & 1.00 & Ref. & 1.00 & Ref. & & 1.00 & Ref. & 1.00 & Ref. & \\
\hline One or more children (aged 0-13 years) & 0.99 & $0.70,1.39$ & 0.93 & $0.58,1.47$ & & 1.26 & $0.92,1.74$ & 0.99 & $0.66,1.47$ & & 0.65 & $0.45,0.95$ & 0.74 & $0.45,1.22$ & \\
\hline One or more adolescents (aged $14-18$ years) & 0.57 & $0.34,0.95$ & 0.76 & $0.38,1.53$ & & 0.91 & $0.54,1.53$ & 0.89 & $0.47,1.70$ & & 1.01 & $0.55,1.86$ & 0.89 & $0.38,2 \cdot 10$ & \\
\hline Children of both age categories & 0.94 & $0.48,1.85$ & 0.98 & $0.40,2.41$ & 0.55 & 0.86 & $0.48,1.55$ & 0.58 & $0.26,1.30$ & 0.55 & 0.77 & $0.38,1.56$ & 0.90 & $0.35,2.32$ & 0.48 \\
\hline \multicolumn{16}{|l|}{ Sweetened beverage consumption } \\
\hline Tertile 1 & 1.00 & Ref. & 1.00 & Ref. & & 1.00 & Ref. & 1.00 & Ref. & & 1.00 & Ref. & 1.00 & Ref. & \\
\hline Tertile 2 & 1.16 & $0.88,1.54$ & 1.15 & $0.80,1.65$ & & 0.91 & $0.70,1.18$ & 0.83 & $0.61,1.14$ & & 1.15 & $0.84,1.58$ & 1.06 & $0.69,1.62$ & \\
\hline Tertile 3 & 1.29 & $0.97,1.71$ & 1.08 & $0.75,1.57$ & 0.40 & 1.20 & $0.92,1.57$ & 1.09 & $0.79,1.50$ & 0.23 & 1.03 & $0.75,1.41$ & $1 \cdot 10$ & $0.73,1.67$ & 0.81 \\
\hline \multicolumn{16}{|l|}{ Artificially sweetened beverages } \\
\hline Non-consumer & 1.00 & Ref. & 1.00 & Ref. & & 1.00 & Ref. & 1.00 & Ref. & & 1.00 & Ref. & 1.00 & Ref. & \\
\hline Consumer & 1.03 & $0.80,1.33$ & 0.92 & $0.66,1.29$ & 0.73 & 0.95 & $0.75,1.20$ & 0.96 & $0.72,1.27$ & 0.90 & 1.02 & $0.77,1.36$ & 1.02 & $0.70,1.49$ & 0.99 \\
\hline
\end{tabular}

Ref., referent category.

Results from multivariable multinomial regression with all variables included in the model. 
Table 5 Multivariable associations between sociodemographic and dietary variables and sugar-sweetened beverage tax perception among a nationally representative sub-sample (largest sample $n$ 1996) from the Nutrinet-Santé cohort study, France, 2012: perception of the health impact variables

\begin{tabular}{|c|c|c|c|c|c|}
\hline & \multicolumn{5}{|c|}{ Having a tax on sweetened beverages would help improve population health $(n 1995)$} \\
\hline & \multicolumn{2}{|c|}{ Agree } & \multicolumn{2}{|c|}{ Neither } & \multirow[b]{2}{*}{$P$} \\
\hline & OR & $95 \% \mathrm{Cl}$ & OR & $95 \% \mathrm{Cl}$ & \\
\hline \multicolumn{6}{|l|}{ Sex } \\
\hline Women & 1.00 & Ref. & 1.00 & Ref. & \\
\hline Men & $1 \cdot 10$ & $0.89,1.37$ & 1.16 & $0.87,1.54$ & 0.557 \\
\hline \multicolumn{6}{|l|}{ Age } \\
\hline $18-25$ years & 0.89 & $0.45,1.78$ & 1.12 & $0.48,2.59$ & \\
\hline $26-45$ years & 1.00 & Ref. & 1.00 & Ref. & \\
\hline $46-65$ years & 1.31 & $1.00,1.73$ & $1 \cdot 16$ & $0.80,1.67$ & \\
\hline$>65$ years & 3.01 & $2.00,4.52$ & 1.63 & $0.96,2.78$ & $<0.001$ \\
\hline \multicolumn{6}{|l|}{ Level of education } \\
\hline No diploma and primary & 0.47 & $0.28,0.78$ & 0.79 & $0.41,1.51$ & \\
\hline Secondary & 0.71 & $0.56,0.90$ & 1.14 & $0.83,1.55$ & \\
\hline Post-secondary graduate & 1.00 & Ref. & 1.00 & Ref. & 0.0004 \\
\hline \multicolumn{6}{|l|}{ Income per household unit } \\
\hline$<900 € /$ month & 0.98 & $0.66,1.44$ & 1.03 & $0.63,1.69$ & \\
\hline $900-2700 € /$ month & 1.00 & Ref. & 1.00 & Ref. & \\
\hline$>2700 € /$ month & $1 \cdot 21$ & $0.91,1.62$ & 0.89 & $0.60,1.31$ & \\
\hline Refuses to answer & 0.99 & $0.68,1.44$ & 0.99 & $0.61,1.60$ & 0.6092 \\
\hline \multicolumn{6}{|l|}{ Household composition } \\
\hline No children & 1.00 & Ref. & 1.00 & Ref. & \\
\hline One or more children (aged $0-13$ years) & 0.72 & $0.53,0.97$ & 0.59 & $0.38,0.89$ & \\
\hline One or more adolescents (aged $14-18$ years) & 0.89 & $0.53,1.50$ & 0.81 & $0.41,1.62$ & \\
\hline Children of both age categories & 0.99 & $0.53,1.86$ & 1.18 & $0.55,2.53$ & 0.2062 \\
\hline \multicolumn{6}{|l|}{ Sweetened beverage consumption } \\
\hline Tertile 1 & 1.00 & Ref. & 1.00 & Ref. & \\
\hline Tertile 2 & 0.75 & $0.58,0.99$ & 0.90 & $0.63,1.28$ & \\
\hline Tertile 3 & 0.67 & $0.51,0.88$ & 0.76 & $0.53,1.09$ & 0.0546 \\
\hline \multicolumn{6}{|l|}{ Artificially sweetened beverages } \\
\hline Non-consumer & 1.00 & Ref. & 1.00 & Ref. & \\
\hline Consumer & 0.87 & $0.69,1.09$ & 0.93 & $0.69,1.27$ & 0.4723 \\
\hline
\end{tabular}

Ref., referent category.

Results from multivariable multinomial regression with all variables included in the model.

obesity rates (e.g. only $19 \%$ of the surveyed individuals estimated that a tax on soft drinks would reduce obesity rates $)^{(33)}$. In comparison, polls conducted in the USA on the public acceptance of a similar tax on sugar-sweetened beverages demonstrated rates of acceptance consistent with our results, i.e. between 37 and $72 \%$, the latter being obtained when respondents were told that the revenue would be used to prevent obesity ${ }^{(32)}$. A telephone survey with a relatively small, nationally representative US sample ( $n$ 592) about perceptions of a sugar-sweetened beverage tax found $36 \%$ of support for the tax scheme ${ }^{(31)}$.

Public acceptance of food taxes depends largely on the intended objectives of the tax. For example, raising funds for public health is generally likely to elicit increased public support ${ }^{(32,34)}$. This is consistent with our results, as participants were more likely to support a tax on unhealthy foods if the generated revenue would be used to improve the health-care system, across sociodemographic variables. It is important to note that the French 'sugar-sweetened beverage tax' was initially conceived as a public health measure to prevent obesity, targeting only sugar-sweetened beverages. However, over time it was re-defined by politicians as a revenue-raising measure, following debates by beverage industry leaders ${ }^{(34)}$. This, in turn, led to the eventual incorporation of artificially sweetened products in the tax definition. This might also explain in part the fact that, in our study, consumers of artificially sweetened beverages were less likely to support the tax scheme.

However, our results showed that participants perceived this taxation scheme as a public health tool targeting unhealthy dietary behaviours, although soft drink consumers were less likely to believe in its efficiency in improving health. The nutrition environment in France, with a national public health nutrition programme in place since 2000, may also contribute to the public's awareness of nutritional matters ${ }^{(35)}$.

Favourable perception of the tax depended on sociodemographic variables, especially age. This could be interpreted in light of two factors: (i) older individuals are less likely to be consumers of soft drinks and therefore might feel less concerned with a taxing scheme on such products than young adults, who are among the heaviest consumers $^{(36)}$; and (ii) older individuals are probably more health conscious ${ }^{(37)}$ and would therefore be more likely to agree with public health prevention measures. Yet, our results are not consistent with those of Rivard et al. who 
observed that younger respondents were more supportive of a sugar-sweetened beverage tax in the USA than were their older counterparts ${ }^{(31)}$.

Next, educational level appeared as an important modulator of tax acceptance, as participants with lower educational levels, unlike more educated participants, were less likely to support the taxation scheme or its application to other unhealthy foods, and also more likely to view the tax as an unfair measure. Our initial hypothesis was that income rather than educational level would be associated with such perceptions, as the potential impact of the measure would be higher in low-income households. During the debates preceding the introduction of the tax, media coverage was rather unfavourable, underlining its potential negative impact on the economy, its effect on the price of beverages and its unfair nature, using comparable arguments as those used in the USA ${ }^{(38)}$. The influence of such media coverage could in part explain our results. In public health matters, our findings argue for targeted communication efforts with individuals with low educational levels. Our results are consistent with those of Rivard et al., showing that support for a tax on sweetened beverages was higher among respondents with higher $v$. those with lower educational levels ${ }^{(31)}$.

Moreover, unlike our initial hypotheses, sugar-sweetened beverage consumption was not a major correlate of tax acceptance. Sugar-sweetened beverage consumption has been found to be negatively associated with age and income in a Belgian population ${ }^{(39)}$, and our results showed that it is indeed these socio-economic characteristics rather than sugar-sweetened beverage consumption itself which are associated with the sugar-sweetened beverage tax perception.

Strengths of our study include the use of a multifaceted questionnaire on tax perception, constructed by a multidisciplinary team, and the initial use of a randomly selected representative sample of the French population. To date, our study is the first to investigate determinants of food tax perception beyond public opinion polls.

Our study is subject to several limitations. First, our population was drawn from a cohort study focused on nutrition. Included participants are therefore more likely to be aware of nutritional issues. However, dietary habits in the Nutrinet-Santé study tend to be consistent with those in other representative samples of the French population ${ }^{(40,41)}$. Second, we aimed at including a sample representative of the French population; however, the final response rate was less than $50 \%$, with a certain degree of selection bias. As included individuals were older and with higher educational levels than excluded ones, our study probably overestimates the tax acceptance rate, since both age and education were found to be significant determinants of support. Third, the tax perception questionnaire was administered 9 to 15 months after the implementation of the tax. Perceptions of the measure might therefore be attenuated compared with the time immediately following its introduction and the media debates. Moreover, as we have no data on perceptions of the tax before or at the time of its implementation, we cannot infer trends in public perception. Repeated questionnaires on perceptions would allow grasping such longitudinal trends. Finally, our study was conducted in a specific setting, with reference to an existing tax scheme. Generalization of our results to other settings should therefore take into account local perception aspects as well as local taxation levels, which influence policy effectiveness $^{(42)}$.

\section{Conclusion}

The French sweetened beverage tax appeared to be favourably received, especially as regards its potential impact on the health of the population. In addition, acceptance and perception of the tax depended on sociodemographic factors. Such elements should be taken into account when advancing novel public health initiatives and presenting them to the general public.

\section{Acknowledgements}

Acknowledgements: The authors thank Dr Fabien Szabo for data management, data cleaning and statistical analyses. They also thank Dr Valentina Andreeva for editing the manuscript. The authors are grateful to the multidisciplinary team involved in the development of the questionnaire: Fabrice Etilé, Nicole Darmon, Céline Bonnet, Bernard Ruffieux, Brice Lepetit and Michel Chauliac. They thank Gwenael Monot, Paul Flanzy, Mohand Ait Oufella, Yasmina Chelghoum, Than Duong Van (computer scientists), Florence Charpentier (dietitian), Anne-Sylvie Monot-Berroyer (communications assistant), Nathalie Arnault, Veronique Gourlet, Fabien Szabo, Laurent Bourhis, Stephen Besseau (statisticians) and Rachida Mehroug (logistics assistant) for their technical contribution to the Nutrinet-Sante study, and all dedicated and conscientious volunteers. Financial support: The present study was funded by the Direction Générale de la Santé. Moreover, the Nutrinet-Santé Study is supported by the French Ministry of Health; the Institut de Veille Sanitaire; the Institut National de la Santé et de la Recherche Médicale; the Institut National de la Recherche Agronomique; the Conservatoire National des Arts et Métiers; the Institut National de Prévention et d'Education pour la Santé; the Fondation pour la Recherche Médicale; and Paris 13 University. Study sponsors had no part in study design, collection, analysis and interpretation of the data, the writing of the article or the decision to submit it for publication. Conflict of interest: None. The researchers are independent of funders and sponsors. Authorship: All researchers had access to all the data. C.J. developed the questionnaire, wrote the statistical analysis plan, analysed the data, and drafted and revised the paper. She is guarantor. 
C.M. developed the questionnaire, participated in the statistical analysis plan, analysed the data and critically revised the paper for important intellectual content. F.V. analysed the data and critically revised the paper for important intellectual content. S.P. developed the questionnaire, analysed the data and critically revised the paper for important intellectual content. S.H. designed the data collection tools, implemented the study, monitored data collection for the whole study, and critically revised the paper for important intellectual content. All authors approved the final version of the manuscript. Ethics of buman subject participation: The Nutrinet-Santé study is conducted in accordance with the Declaration of Helsinki and all procedures have been approved by the International Research Board of the French Institute for Health and Medical Research (0000388FWA00005831) and the Commission Nationale Informatique et Libertés (908450 and 909216). Electronic informed consent was obtained from all participants.

\section{Supplementary material}

To view supplementary material for this article, please visit http://dx.doi.org/10.1017/S1368980014003231

\section{References}

1. Finucane MM, Stevens GA, Cowan MJ et al. (2011) National, regional, and global trends in body-mass index since 1980: systematic analysis of health examination surveys and epidemiological studies with 960 country-years and 9.1 million participants. Lancet 377, 557-567.

2. Unité de Surveillance et d'Epidemiologie Nutritionnelle (2007) Etude nationale Nutrition Santé (ENNS, 2006). Situation nutritionnelle en France en 2006 selon les indicateurs d'objectifs et les repères du Programme National Nutrition Santé (PNNS). Paris: Institut de Veille Sanitaire, Université Paris 13, Conservatoire National des Arts et Metiers.

3. World Health Organization (2003) Diet, Nutrition and the Prevention of Chronic Diseases. Report of a Joint WHO/FAO Expert Consultation. WHO Technical Report Series no. 916. Geneva: WHO.

4. World Health Organization (2009) Global Health Risks: Mortality and Burden of Disease Attributable to Selected Major Risks. Geneva: WHO.

5. Cecchini M, Sassi F, Lauer JA et al. (2010) Tackling of unhealthy diets, physical inactivity, and obesity: health effects and cost-effectiveness. Lancet 376, 1775-1784.

6. Epstein LH, Jankowiak N, Nederkoorn C et al. (2012) Experimental research on the relation between food price changes and food-purchasing patterns: a targeted review. Am J Clin Nutr 95, 789-809.

7. Eyles H, Ni Mhurchu C, Nghiem N et al. (2012) Food pricing strategies, population diets, and non-communicable disease: a systematic review of simulation studies. PLoS Med $\mathbf{9}$, e1001353.

8. Franck C, Grandi SM \& Eisenberg MJ (2013) Taxing junk food to counter obesity. Am J Public Health 103, 1949-1953.

9. Green R, Cornelsen L, Dangour AD et al. (2013) The effect of rising food prices on food consumption: systematic review with meta-regression. BMJ 346, f3703.
10. Jacobson MF \& Brownell KD (2000) Small taxes on soft drinks and snack foods to promote health. Am J Public Health 90, 854-857.

11. Jensen JD \& Smed S (2007) Cost-effective design of economic instruments in nutrition policy. Int J Behav Nutr Phys Act 4, 10.

12. Kim D \& Kawachi I (2006) Food taxation and pricing strategies to 'thin out' the obesity epidemic. Am J Prev Med 30, 430-437.

13. Marshall T (2000) Exploring a fiscal food policy: the case of diet and ischaemic heart disease. BMJ 320, 301-304.

14. Mytton OT, Clarke D \& Rayner M (2012) Taxing unhealthy food and drinks to improve health. BMJ 344, e2931.

15. Nnoaham KE, Sacks G, Rayner M et al. (2009) Modelling income group differences in the health and economic impacts of targeted food taxes and subsidies. Int J Epidemiol 38, 1324-1333.

16. Powell LM \& Chaloupka FJ (2009) Food prices and obesity: evidence and policy implications for taxes and subsidies. Milbank Q 87, 229-257.

17. Schroeter C, Lusk J \& Tyner W (2008) Determining the impact of food price and income changes on body weight. $J$ Health Econ 27, 45-68.

18. Smed S, Jensen JD \& Denver S (2007) Socio-economic characteristics and the effect of taxation as a health policy instrument. Food Policy 32, 624-639.

19. Thow AM, Jan S, Leeder S et al. (2010) The effect of fiscal policy on diet, obesity and chronic disease: a systematic review. Bull World Health Organ 88, 609-614.

20. Tiffin R \& Arnoult M (2011) The public health impacts of a fat tax. Eur J Clin Nutr 65, 427-433.

21. Waterlander WE, Steenhuis IHM, de Boer MR et al. (2012) Introducing taxes, subsidies or both: the effects of various food pricing strategies in a web-based supermarket randomized trial. Prev Med 54, 323-330.

22. Cabrera Escobar MA, Veerman JL, Tollman SM et al. (2013) Evidence that a tax on sugar sweetened beverages reduces the obesity rate: a meta-analysis. BMC Public Health 13, 1072.

23. Powell LM, Chriqui JF, Khan T et al. (2013) Assessing the potential effectiveness of food and beverage taxes and subsidies for improving public health: a systematic review of prices, demand and body weight outcomes. Obes Rev 14, $110-128$.

24. Malik VS, Pan A, Willett WC et al. (2013) Sugar-sweetened beverages and weight gain in children and adults: a systematic review and meta-analysis. Am J Clin Nutr 98, 1084-1102.

25. Vartanian LR, Schwartz MB \& Brownell KD (2007) Effects of soft drink consumption on nutrition and health: a systematic review and meta-analysis. Am J Public Health 97, 667-675.

26. James J, Thomas P, Cavan D et al. (2004) Preventing childhood obesity by reducing consumption of carbonated drinks: cluster randomised controlled trial. BMJ 328, 1237-1239.

27. République Française (2014) Code général des impôts, CGI.Article 1613 ter. http://www.legifrance.gouv.fr/affichCode Article.do?idArticle=LEGIARTI000025051331\&cidTexte=LEGIT EXT000006069577 (accessed March 2014).

28. Hercberg S, Castetbon K, Czernichow S et al. (2010) The Nutrinet-Sante Study: a web-based prospective study on the relationship between nutrition and health and determinants of dietary patterns and nutritional status. BMC Public Health 10, 242.

29. Kesse-Guyot E, Castetbon K, Touvier M et al. (2010) Relative validity and reproducibility of a food frequency questionnaire designed for French adults. Ann Nutr Metab 57, 153-162.

30. Institut National de la Statistique et des Etudes Economiques (2012) Méthodes. http://www.insee.fr/en/methodes/ (accessed March 2014). 
31. Rivard C, Smith D, McCann SE et al. (2012) Taxing sugarsweetened beverages: a survey of knowledge, attitudes and behaviours. Public Health Nutr 15, 1355-1361.

32. Brownell KD \& Frieden TR (2009) Ounces of prevention the public policy case for taxes on sugared beverages. $N$ Engl J Med 360, 1805-1808.

33. Levy JD \& Lancrey-Javal G (2011) La réaction des Français suite aux annonces de François Fillon du 24 août 2011. http:// www.harrisinteractive.fr/news/2011/CP_HIFR_economie_ 07092011.pdf (accessed March 2014).

34. Landon J \& Graff H (2012) What is the Role of HealthRelated Food Duties? A report of the National Heart Forum meeting held on 29 June 2012. London: National Heart Forum.

35. Hercberg S, Chat-Yung S \& Chauliac M (2008) The French National Nutrition and Health Program: 2001-2006-2010. Int J Public Health 53, 68-77.

36. Dallongeville J (2012) Consommation de boissons sucrées: relation avec la surcharge pondérale et l'obésité. Cah Nutr Diet 47, 66-71.

37. Kearney JM, Gibney MJ, Livingstone BE et al. (2001) Attitudes towards and beliefs about nutrition and health among a random sample of adults in the Republic of Ireland and Northern Ireland. Public Health Nutr 4, 1117-1126.

38. Niederdeppe J, Gollust SE, Jarlenski MP et al. (2013) News coverage of sugar-sweetened beverage taxes: pro- and antitax arguments in public discourse. Am J Public Health 103, E92-E98.

39. Mullie P, Aerenhouts D \& Clarys P (2012) Demographic, socioeconomic and nutritional determinants of daily versus non-daily sugar-sweetened and artificially sweetened beverage consumption. Eur J Clin Nutr 66, 150-155.

40. Castetbon K, Vernay M, Malon A et al. (2009) Dietary intake, physical activity and nutritional status in adults: the French nutrition and health survey (ENNS, 2006-2007). Br J Nutr 102, 733-743.

41. Lassale C, Galan P, Julia C et al. (2013) Association between adherence to nutritional guidelines, the metabolic syndrome and adiposity markers in a French adult general population. PLoS One 8, e76349.

42. Jou J \& Techakehakij W (2012) International application of sugar-sweetened beverage (SSB) taxation in obesity reduction: factors that may influence policy effectiveness in countryspecific contexts. Health Policy 107, 83-90. 